\title{
Characterization of End Groups in Polycarbonates by Reactive Pyrolysis-Gas Chromatography
}

\author{
Yoshiro Ito, Hiromi Ogasawara, Yasuyuki Ishida, \\ Hajime OHTANI, ${ }^{*}$ and Shin Tsuge ${ }^{\dagger}$ \\ Department of Applied Chemistry, School of Engineering, \\ Nagoya University, Nagoya 464-01, Japan \\ * Division of Materials Science, Center for Integrated Research in Science \\ and Engineering, Nagoya University, Nagoya 464-01, Japan
}

(Received June 14, 1996)

\begin{abstract}
Pyrolysis-gas chromatography using the sample decomposition in the presence of an organic alkali, tetramethylammonium hydroxide (TMAH), was applied for end group determination of industrially available polycarbonate (PC)'s. Various phenolic compounds were observed in the conventional pyrograms of the PC's, which were formed through the $\mathrm{C}-\mathrm{C}$ bond cleavage as well as that of carbonate linkages. On the other hand, the PC main chain almost quantitatively degraded through reactive pyrolysis at carbonate linkages to yield the dimethyl derivatives of the constituents such as bisphenol-A through a hydrolytic pyrolysis reaction in the presence of TMAH. In a similar manner, characteristic meth$\mathrm{yl}$ ethers such as anisole and p-tert-butylanisole were formed from end groups. By this method, highly accurate and rapid determination became possible for the contents of their end groups. This information, in turn, enabled accurate estimation of the number average molecular weight of PC's without using any reference polymers.

KEY WORDS Pyrolysis-Gas Chromatography / Polycarbonate / End Group / Tetramethylammonium Hydroxide / Number-Average Molecular Weight /
\end{abstract}

It is well known that the structure of end groups changes depending upon the polymerization processes, such as initiation, chain transfer, and termination mechanisms. Furthermore, since initiators and/or termination reagents for polymerization incorporated into the polymer chain ends often cause significant changes in properties of the resulting polymer. This has led to a growing demand for accurate and precise characterization of end groups. However, end group characterization is not an easy task because of their very low concentration compared with monomeric ones which constitute the main chain of the polymer.

Recently, nuclear magnetic resonance (NMR) has been extensively utilized to characterize end groups of polymers. ${ }^{1}$ In addition, pyrolysis-gas chromatography (PyGC) has been noted as a very powerful tool to analyze the end groups in poly(methyl methacrylate), ${ }^{2-7}$ polystyrene (PS), ${ }^{8,9}$ and the others. ${ }^{10}$

With respect to polycarbonate (PC), most of the literatures describing end groups dealt with uncapped oligomers produced through hydrolysis of the polymer. Techniques used to characterize phenolic end group in uncapped PC oligomers includes ultraviolet spectrometry, ${ }^{11-13}$ potentiometric titration, ${ }^{14}$ infrared spectrometry, ${ }^{12}$ and size exclusion chromatography (SEC). ${ }^{12,13}$ Although conventional Py-GC was also used to characterize the end groups in PC's, the reported results were restricted to the identification and semi-quantitative estimation of average molecular weight $(M W)$ of fractionated polycarbonate (PC)'s, ${ }^{15}$ mainly because of the less quantitative recoveries of the degradation products on the pyrogram leaving some residue after pyrolysis and the complexity of the products consisting of various polar phenolic compounds.

Recently, Challinor ${ }^{16}$ reported that pyrolysis of vari-

+ To whom correspondence should be addressed. ous condensation polymers in the presence of organic alkali such as tetramethylammonium hydroxide (TMAH) resulted in a simplified pyrogram consisting of methyl derivative peaks which are quantitatively formed from the constituents of the polymer. Applying this technique, the compositional analysis of aromatic polyesters ${ }^{17}$ and the quantitative determination of methacryloyl end groups of PS macromonomer ${ }^{7}$ were successfully achieved on the basis of the observed pyrograms. The same technique has been also applied in the fields of characterizing microorganisms, geological samples and rosin sizing agents in paper. ${ }^{18-21}$

In this work, reactive Py-GC in the presence of TMAH is applied for quantitative characterization of the end groups in industrially available PC's. Results obtained by this method are compared with those by SEC and ${ }^{1} \mathrm{H}$ NMR.

\section{EXPERIMENTAL}

\section{Samples}

Poly-[2,2-propane-bis(4-phenyl carbonate)] (PC) is one of the most important engineering plastics bearing excellent transparency and mechanical properties. The main chain of ordinary PC molecules consist of bisphenol-A units bonded with carbonate linkages, while various terminal groups are formed depending on the polymerization process. The solvent method (SM) in which $p$-tert-butylphenol is often used to control the $M W$ of the polymers in addition to the main materials such as sodium salt of bisphenol-A and phosgen, provides PC molecules endcapped with $p$-tert-butylphenoxy groups as shown in Scheme 1. On the other hand, the melt method (MM), in which bisphenol-A and diphenylcarbonate are directly reacted through transesterification, is known to yield PC molecules with either phenoxy or hydroxyl end group described in Scheme 2. 

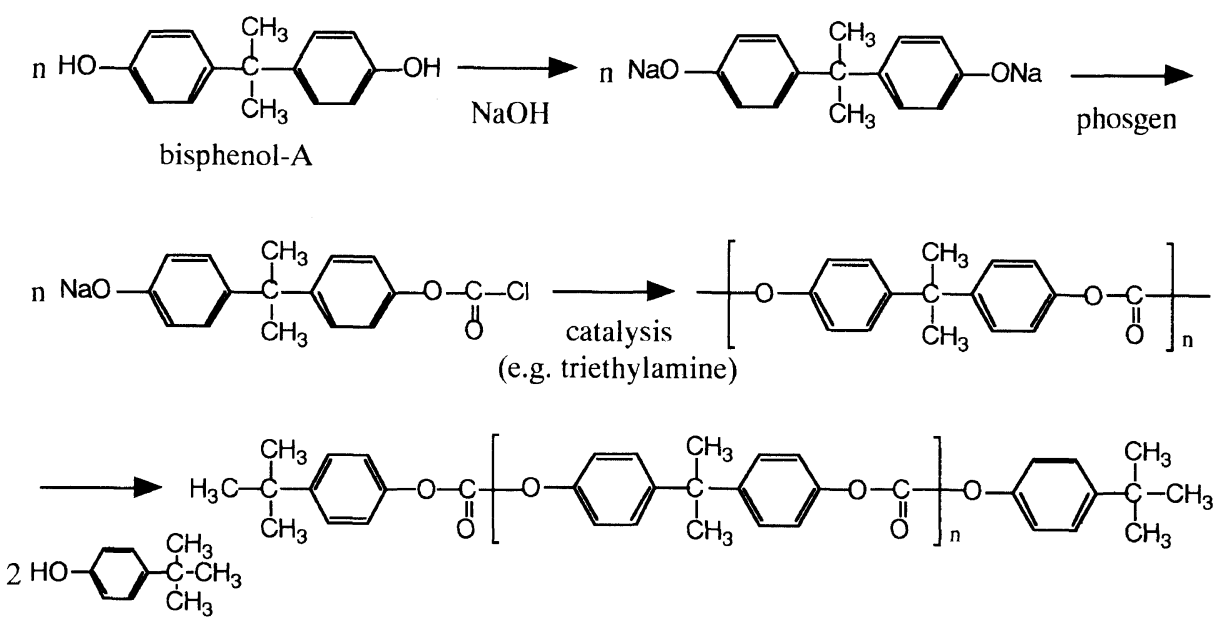

Scheme 1. Solvent method.
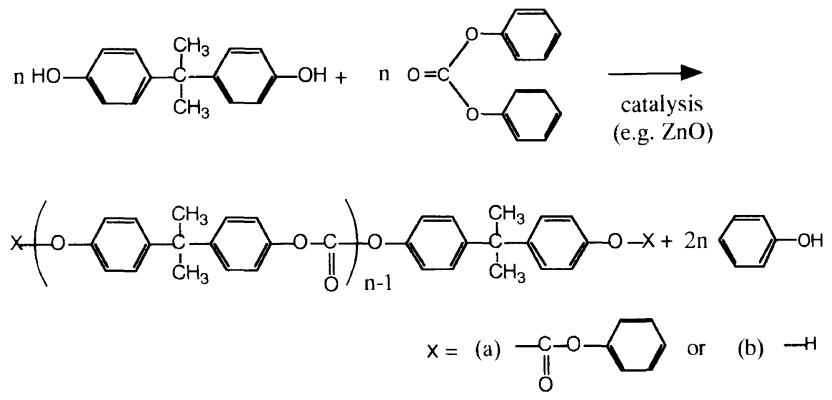

Scheme 2. Melt method (transesterification).

\section{Sample Preparation}

In this work, industrially available SM- and MM-PC samples were fractionated as follows. Raw PC weighing about $10 \mathrm{~g}$ was dissolved in $500 \mathrm{ml}$ of methylene chloride. After an aliquot of precipitant ( $n$-heptane) was added to the solution, the resulting precipitate was filtrated off as the first fraction. This procedure was repeated for the filtrate. In this manner, the original two solutions were fractionated into three fractions $\mathrm{M}-1-\mathrm{M}-3$ and $\mathrm{S}-1-$ $\mathrm{S}-3$, respectively by the successive reprecipitation method both for MM- and SM-PC samples. Each fraction was dried in vacuo at $60^{\circ} \mathrm{C}$ for 24 hours.

\section{Reactive Py-GC Conditions}

Figure 1 illustrates the sample preparation for reactive pyrolysis. A vertical micro furnace pyrolyzer (Yanaco GP-1028) was directly attached to the injection port of a gas chromatograph (Shimadzu 7AG) equipped with a flame ionization detector (FID). About $50 \mu \mathrm{g}$ of the cryomilled PC powder and $1 \mu \mathrm{l}$ of TMAH solution ( $25 \mathrm{wt} \%$ in methanol) (Aldrich Chemical Co., Inc.) taken in a platinum sample cup was introduced into the heated center of the pyrolyzer under the flow of He carrier gas. In this study, the hydrolytic pyrolysis temperature in the presence of TMAH was empirically set at $400^{\circ} \mathrm{C}$ because of the following reason. Above $400^{\circ} \mathrm{C}$ additional peaks were produced mainly due to the contribution of the ordinary pyrolysis, while bellow $400^{\circ} \mathrm{C}$ significant peak broadening was observed. A fused silica capillary column (Quadrex MS, $25 \mathrm{~m}$ long $\times 0.25 \mathrm{~mm}$ i.d.) coated with polydimethylsiloxane $(0.25 \mu \mathrm{m}$ thick $)$ was used. The $50 \mathrm{ml} \mathrm{min}^{-1}$ carrier gas flow rate at the pyrolyzer was

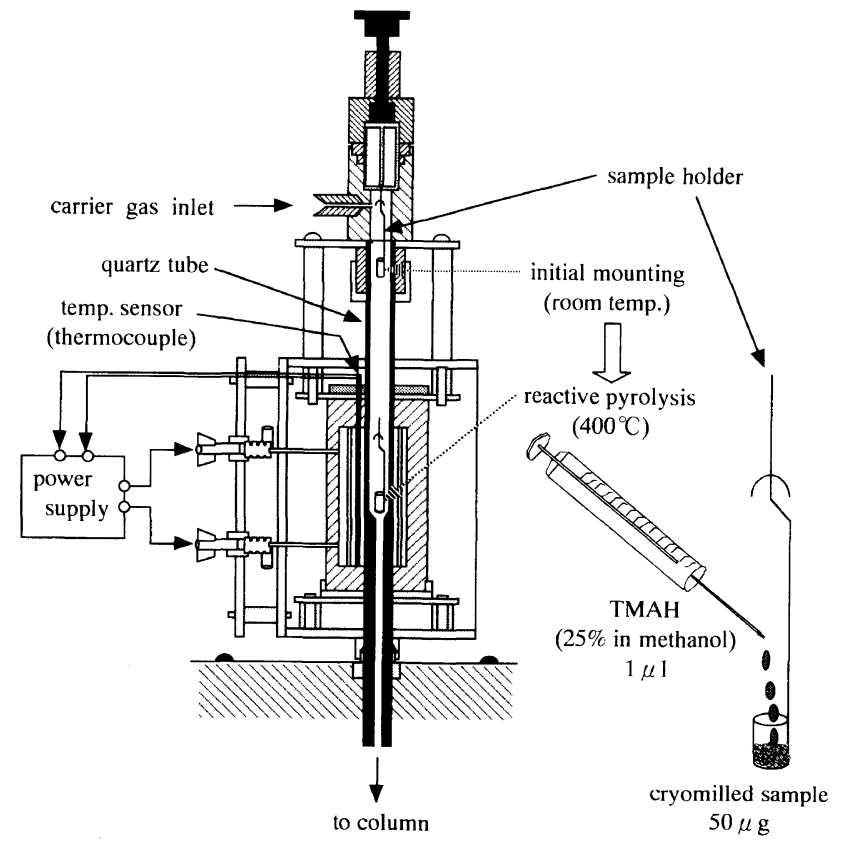

Figure 1. Vertical micro furnace pyrolyzer and sample preparaton.

reduced to $1.3 \mathrm{ml} \mathrm{min}^{-1}$ at the capillary column by means of a splitter. The column temperature was initially set at $50^{\circ} \mathrm{C}$ and then temperature programmed up to $300^{\circ} \mathrm{C}$ at a rate of $4^{\circ} \mathrm{Cmin}^{-1}$. The identification of the characteristic peaks on the resulting pyrogram was mostly carried out by use of a GC/mass spectrometer (MS) system (Shimadzu QP1000) to which the pyrolyzer was also attached.

\section{SEC Measurement}

The average $M W$ of each fractionated $\mathrm{PC}$ was measured by SEC using PS standards. The system consisted of a column oven (Japan Spectroscopic Co., Ltd. [JASCO] 860-CO), a pump (JASCO 800-PU), a refractive index detector (JASCO 830-RI) and a integrator (JASCO 807-IT). A $100 \mu \mathrm{l}$ sample solution $(0.25 \%)$ was injected into a column (Showa Denko K. K., Shodex K-803 combined in series with K-80M) held at $30^{\circ} \mathrm{C}$ under a $1 \mathrm{ml} \mathrm{min}^{-1}$ flow of chloroform.

The intrinsic viscosity $([\eta])$ of a given polymer is given by Mark-Houwink-Sakurada's equation, 


$$
[\eta]=K M^{a}
$$

where $M$ is $M W$ of a polymer, and $K$ and $a$ are the specific constants characteristic of the polymer system under given solvent and temperature. In SEC measurements, the relationship between $[\eta]$ and $M$ of different polymers eluting at the same retention volume is given by

$$
[\eta]_{1} M_{1}=[\eta]_{2} M_{2}
$$

where $[\eta]_{1}$ and $M_{1}$ are values for a given polymer, and $[\eta]_{2}$ and $M_{2}$ are those for the reference polymer. Provided that $M_{1}$ represents the actual $M W$ of a given PC sample and $M_{2}$ is that of PS standard eluting at the same retention volume, $M_{1}$ can be determined by substituting Mark-Houwink-Sakurada's viscosity equation into equation 2 and arranging,

$$
\log M_{1}=\frac{1}{a_{1}+1} \log \frac{K_{2}}{K_{1}}+\frac{a_{1}+1}{a_{1}+1} \log M_{2}
$$

(a)

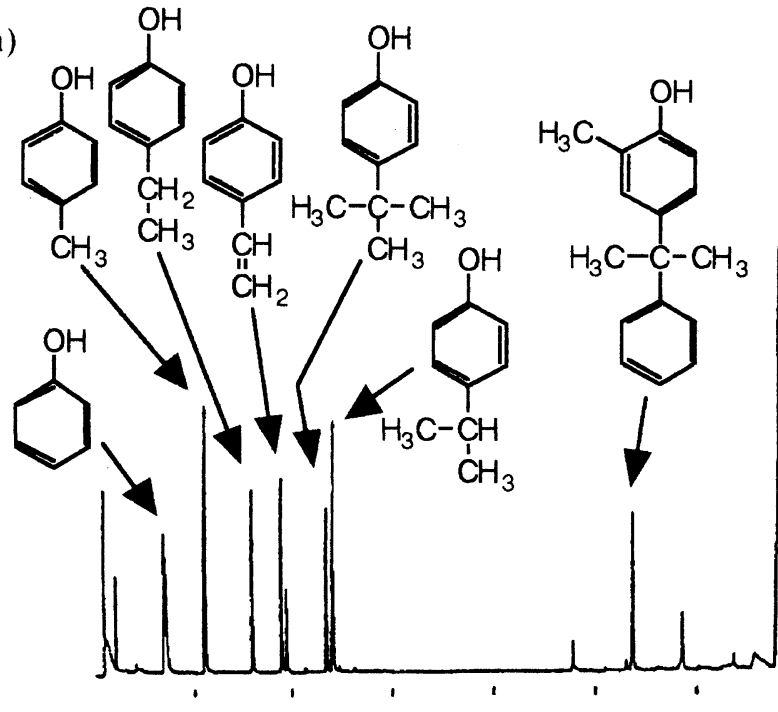

10

20

(b)

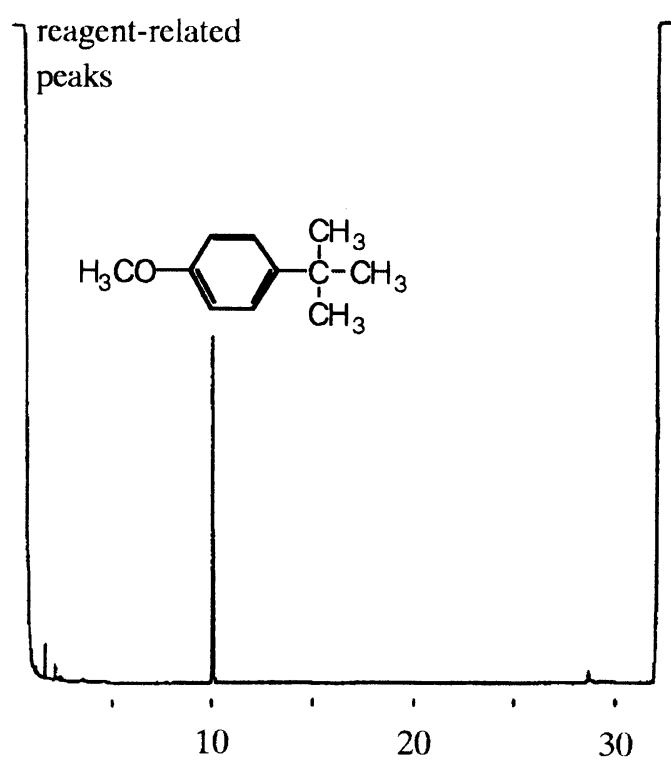

where $a_{1}=0.82$ and $K_{1}=1.20 \times 10^{-4}$ for PC in chloroform at $25^{\circ} \mathrm{C}$, and $a_{2}=0.794$ and $K_{2}=0.49 \times 10^{-4}$ for PS in chloroform at $30^{\circ} \mathrm{C}$ available from references were used. ${ }^{22,23}$

\section{NMR Measurement}

${ }^{1} \mathrm{H}$ NMR spectra were obtained on a Varian VXR500 $(500 \mathrm{MHz})$ spectrometer under the following conditions; spectral width of $8000 \mathrm{~Hz}$, acquisition time of $2 \mathrm{~s}$, relaxation time of $3 \mathrm{~s}$ and pulse width of $1.0 \mu \mathrm{s}$. About $5 \mathrm{mg}$ of a sample dissolved in $\mathrm{CDCl}_{3}(\mathrm{ca} .1 \mathrm{ml})$ was measured at room temperature. Chemical sifts were recorded in parts per million relative to the standard tetramethylsilane. The accumulation of 100 scans (about $10 \mathrm{~min}$ ) was used to obtain ${ }^{1} \mathrm{H}$ NMR spectra having sufficient $\mathrm{S} / \mathrm{N}$ values for each samples.

\section{RESULTS AND DISCUSSION}

Figure 2 shows the typical pyrograms of (a) a raw 
SM-PC sample observed by conventional pyrolysis at $600^{\circ} \mathrm{C}$, and (b) a fractionated SM-PC (S-2) observed by reactive pyrolysis at $400^{\circ} \mathrm{C}$ in the presence of TMAH. In the case of conventional pyrolysis at $600^{\circ} \mathrm{C}$, various phenolic compounds such as phenol, cresols and bisphenol-A were formed through the cleavages not only at carbonate linkages but also at $\mathrm{C}-\mathrm{C}$ bondings. ${ }^{15,24-28}$ Although p-tert-butylphenol is observed as one of the characteristic products from the terminal groups, the quantitative analysis of the end group is not possible by using the relative peak intensity of $p$-tert-butylphenol, because of less quantitative recovery on the observed pyrogram.

When the SM-PC sample was pyrolyzed at $400^{\circ} \mathrm{C}$ without adding TMAH, only small and broad peaks were observed because of slow and insufficient thermal decomposition. In contrast, using reactive pyrolysis at $400^{\circ} \mathrm{C}$ in the presence of $\mathrm{TMAH}$, the $\mathrm{PC}$ decompose selectively at the carbonate linkages into dimethylether of bisphenol-A from the main chain along with $p$ tert-butylanisole from the terminal groups. Thus, after the excess reagent-related products such as trimethyl amine and methanol are eluted, only the two main peaks are observed in the pyrogram; p-tert-butylanisole and dimethylether of bisphenol-A. In this case, the whole polymer samples were almost quantitatively degraded into these two products. Therefore, the content of end groups can be accurately determined from the relative peak areas between these two peaks after making the molar sensitivity correction for the FID response by use of the effective carbon number. ${ }^{29}$

Here, since both terminals in the SM-PC molecules are supposed to be completely end-capped with $p$-tertbutylphenoxy groups, the number average molecular weight $\left(M_{n}\right)$ of the SM-PC samples can be estimated from the result of the end group determination as follows,

$$
\begin{gathered}
D P=\frac{I_{\mathrm{M}} / 15.4}{\left(I_{E} / 10.2\right) / 2} \\
M_{n}=D P \times 254+326
\end{gathered}
$$

where $D P$ is the degree of polymerization, $I_{\mathrm{M}}$ and $I_{\mathrm{E}}$ are the intensities of the peaks due to the main chain and the end groups, respectively, and divisors 15.4 and 10.2 are empirically determined effective carbon numbers, which compensated the molar sensitivity for FID response, of the respective components. Since one polymer molecule has two end groups, the denominator in eq 4 was divided by 2 . The values of 254 and 326 in eq 5 are the $M W$ of the monomer unit and the two end groups, respectively.

Figure 3 shows a methyl-proton region of the ${ }^{1} \mathrm{H}$ NMR spectrum of a fractionated SM-PC (S-2). Peaks appeared at $1.68 \mathrm{ppm}$ and $1.32 \mathrm{ppm}$ were assigned to methylprotons of bisphenol-A and those of the end groups, that is, the $p$-tert-butylphenoxy groups, respectively. The end groups and then $M_{n}$ are also determined using relative intensities between these two peaks.

Estimated $M_{n}$ values by reactive Py-GC for three fractions of SM-PC are summarized in Table I along with those by SEC and NMR. Generally, the values determined by Py-GC are in fairly good agreement with those obtained by NMR. However, those by SEC show slightly smaller values. Considering the fact that the $M_{n}$ values by SEC are calculated empirically using PS standards, those by Py-GC and NMR, in which any reference standards are not used, might be rather close

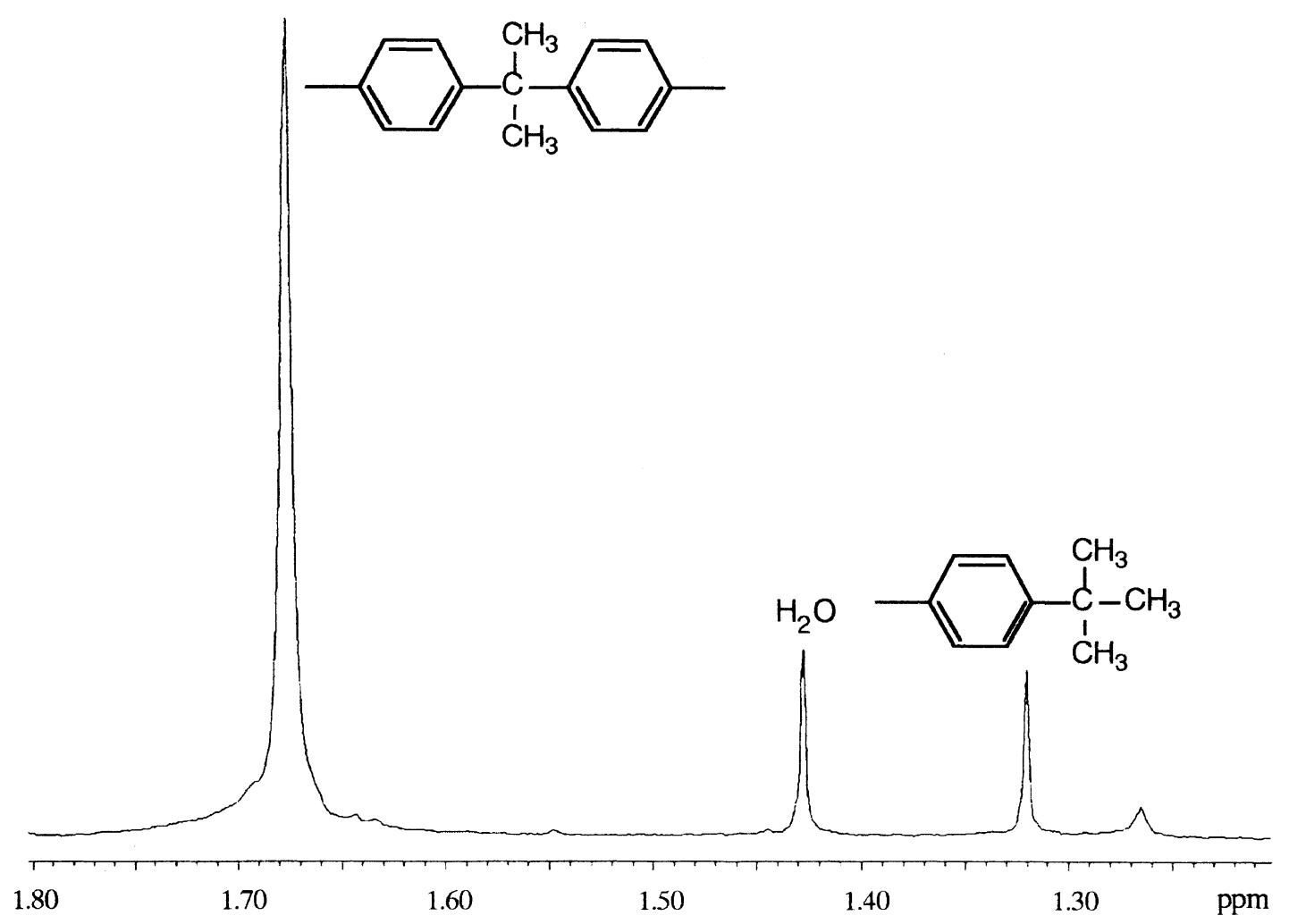

Figure 3 Methyl-proton region of ${ }^{1} \mathrm{H}$ NMR spectrum of SM-PC (S-2) measured in $\mathrm{CDCl}_{3}$ at room temperature. Peaks at 1.78 ppm and $1.32 \mathrm{ppm}$ are due to a bisphenol-A (monomer unit) and the end group, respectively. Peak at 1.43 ppm is due to $\mathrm{H}_{2} \mathrm{O}$ mainly contaminated in $\mathrm{CDCl}_{3}$. 
to the absolute ones. Furthermore, highly precise results can be obtained by Py-GC, e.g., $C V=1.8 \%$ for $\mathrm{S}-2$ and $0.6 \%$ for S-3 sample for five repeated measurements.

Figure 4 shows the typical pyrograms of (a) a raw MM-PC sample observed by conventional pyrolysis at $600^{\circ} \mathrm{C}$, and (b) a fractionated MM-PC (M-1) observed by reactive pyrolysis at $400^{\circ} \mathrm{C}$ in the presence of TMAH. Similarly to the case of SM-PC, various phenolic compounds were observed in the pyrogram (a). In this

Table I. Estimated number average molecular weight of fractionated SM-PC sample

\begin{tabular}{cccc}
\hline & \multicolumn{3}{c}{ Estimated $M_{n}$ values } \\
\cline { 2 - 4 } Fraction no. & by Py-GC & by NMR & by SEC \\
\hline S-1 & 27200 & 30200 & 23400 \\
S-2 & $9800^{\text {a }}$ & 9600 & 8300 \\
S-3 & $5000^{\text {b }}$ & - & 4000 \\
\hline
\end{tabular}

${ }^{\mathrm{a}} C V=1.8 \%$ for five measurements. ${ }^{\mathrm{b}} \mathrm{CV}=0.6 \%$. case, the characteristic products of the end groups in the MM-PC molecules are to be phenol and bisphenol-A from the phenoxy and the hydroxyl end groups, respectively. However, the fact that phenol is also observed in Figure 2(a) indicates that the characteristic products of the end groups are indistinguishable from those formed from the main chain in the MM-PC samples. Therefore, the end groups in MM-PC's can not be directly determined by conventional Py-GC. In addition, as for NMR measurement for MM-PC samples, we can not obtain quantitative results for the end group information, because it is not possible to distinguish proton signals due to end phenyl and hydroxyphenyl group from those due to phenyl groups in the main chain of PC.

On the other hand, a peak of anisole, which is the characteristic product from the terminal phenoxy group, is completely separated from that of dimethyl ether of bisphenol-A formed from the main chain in Figure 4(b). Therefore, the content of phenoxy terminal groups can
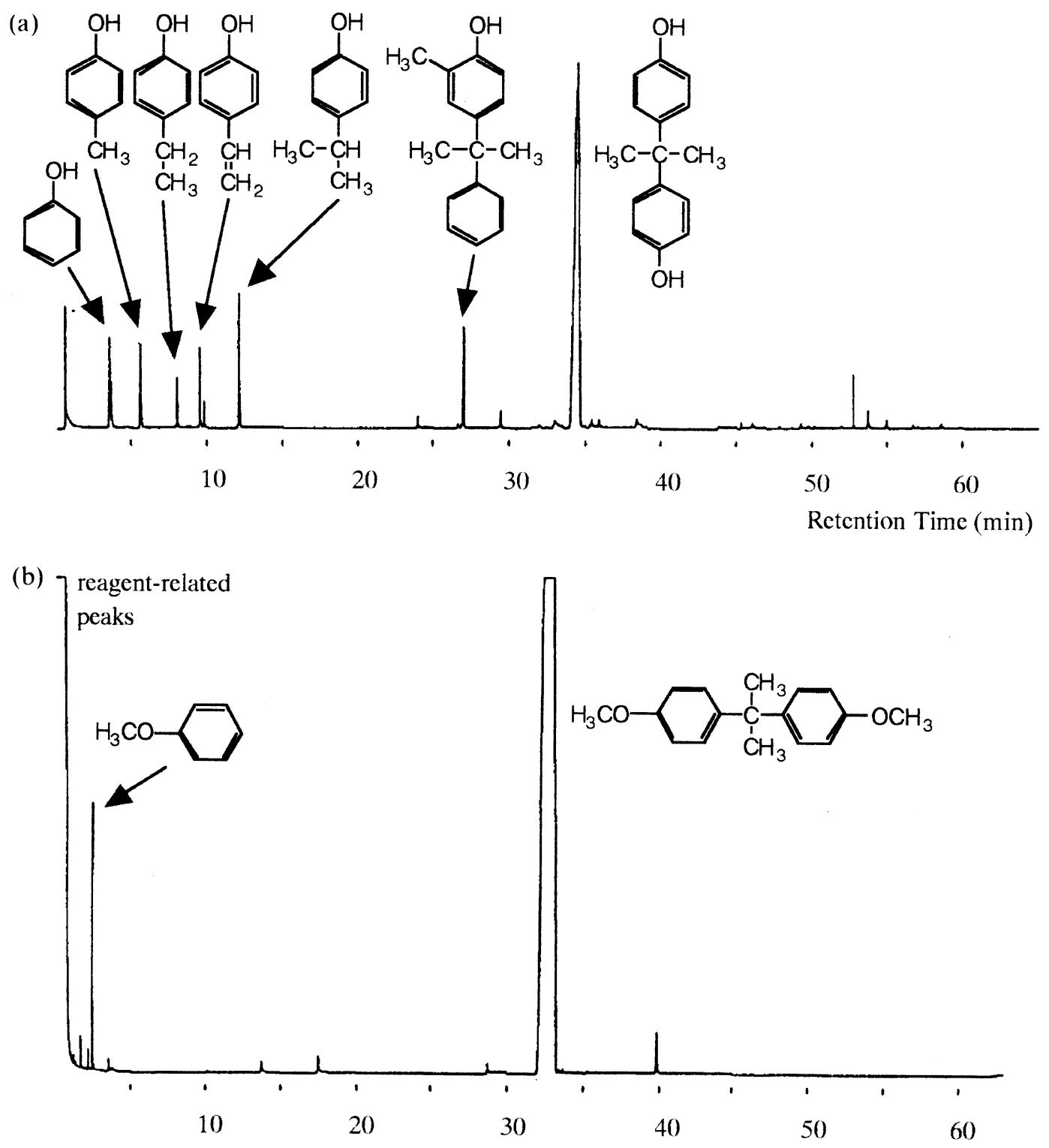

Retention Time (min)

Figure 4. Pyrograms of MM-PC: (a) a raw MM-PC observed by conventional pyrolysis at $600^{\circ} \mathrm{C}$ and (b) a fractionated MM-PC (M-1) observed by reactive pyrolysis at $400^{\circ} \mathrm{C}$ in the presence of TMAH. 
Table II. Average number of phenoxy end groups in a fractionated MM-PC molecule

\begin{tabular}{ccccc}
\hline Fraction no. & $M_{n}{ }^{\mathrm{a}}$ & $\begin{array}{c}n / a^{\mathrm{b}} \\
\text { by Py-GC }\end{array}$ & $\% a^{\mathrm{c}}$ & $\% b^{\mathrm{d}}$ \\
\hline M-1 & 34300 & 94.2 & 71.4 & 28.6 \\
M-2 & 16300 & 38.0 & 83.6 & 16.4 \\
M-3 & 4200 & 11.9 & 67.4 & 32.6 \\
\hline
\end{tabular}

${ }^{a}$ Corrected after SEC measurement. $\quad{ }^{b}$ The ratio between monomer units and phenoxy end groups. ${ }^{\mathrm{c}}$ Proportion of phenoxy end group in a molecule $(\% a+\% b=100)$. $\quad{ }^{d}$ Proportion of hydroxyphenyl end group in a molecule.

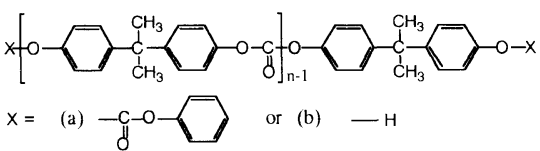

be determined from the peak area ratio between anisole and dimethylether of bisphenol-A. The other minor unknown peaks were not taken into consideration because of their negligibly small peak intensities.

In the case of the MM-PC, it is known that the numbers of phenoxy and hydroxyl end groups are not always the same, but changes depending on the polymerization conditions. Here, once the ratio between monomer units and phenoxy end groups $(n / a)$, and $M_{n}$ are determined, the proportion of each end group can be estimated. Table II summarizes the proportion of phenoxy end group $(\% a)$ in the molecules for each fraction of MM-PC along with their corrected $M_{n}$ and the ratios, $n / a$ obtained by reactive Py-GC. Here the $M_{n}$ values measured by SEC were corrected using the following relation,

$$
\begin{gathered}
M_{n}(\text { by } \mathrm{Py}-\mathrm{GC})=1.15 \times M_{n}(\text { by SEC })+358 \\
\text { correlation factor: } 0.9999
\end{gathered}
$$

where the constants 1.15 and 358 are determined by the least-square method on the basis of the results for SM-PC fractions shown in Table I.

The results in Table II suggest that about $70-80 \%$ of the end groups are phenoxy ones in the given MM-PC samples. It is known that the hydroxyl end groups lower the thermal stabilities of PC. Thus the observed results also suggest that the industrial PC is generally produced under the polymerization condition to lessen the hydroxyl end groups. ${ }^{30}$

\section{CONCLUSION}

Reactive Py-GC in the presence of an organic alkali, TMAH, proved to be a very effective technique to characterize the end groups in PC's. The PC main chain almost quantitatively degraded through reactive pyrolysis into the dimethyl derivatives of bisphenol-A, while the end groups yielded characteristic methyl ether such as anisole and p-tert-butylanisole. By this method, accurate determination of the end groups became possible. Additionally, this technique enabled highly accurate estimation of $M_{n}$ of PC's without using any reference polymers.
Provided that the $\mathrm{S} / \mathrm{N}=2$ for the products characteristic of the end groups is the detectable limit on the resulting pyrograms, even the $M_{n} \geq 10^{6}$ could be estimated for both SM-PC and MM-PC.

Acknowledgments. Financial supports by the Grantin-Aid for Developmental Scientific Research (B) (07555262) of the Ministry of Education, Science, Sports, and Culture of Japan, by the Mazda Foundation, and by the Tatematsu Foundation are greatly acknowledged.

\section{REFERENCES}

1. J. C. Bevington, J. R. Ebdon, and T. N. Huckerby, in "NMR Spectrometry of Polymers," R. N. Ibblet, Ed., Blackie Academic \& Professional, Glasgow, 1993, pp 80-121.

2. H. Ohtani, S. Ishiguro, M. Tanaka, and S. Tsuge, Polym. J., 21, 41 (1989).

3. H. Ohtani, M. Tanaka, and S. Tsuge, J. Anal. Appl. Pyrolysis, 15, 167 (1989).

4. H. Ohtani, M. Tanaka, and S. Tsuge, Bull. Chem. Soc. Jpn., 63, 1196 (1990).

5. Y Tsukahara, Y. Nakanishi, Y. Yamashita, H. Ohtani, Y. Nakashima, Y. F. Luo, T. Ando, and S. Tsuge, Macromolecules, 24, 2493 (1991).

6. H. Ohtani, Y. F. Luo, Y. Nakashima, Y. Tsukahara, and S. Tsuge, Anal. Chem., 66, 1438 (1994).

7. H. Ohtani, S. Ueda, Y. Tsukahara, C. Watanabe, and S. Tsuge, J. Anal. Appl. Pyrolysis, 25, 1 (1993).

8. Y. Ito, S. Tsuge, H. Ohtani, J. Atarashi, S. Wakabayashi, and T. Kawamura, Macromolecules, in press.

9. Y. Ito, H. Ohtani, S. Ueda, Y. Nakashima, and S. Tsuge, J. Polym. Sci., A, 32, 383 (1994).

10. T. Usami, F. Keitoku, H. Ohtani, and S. Tsuge, Polymer, 33, 3024 (1992).

11. H. Shchori and J. E. McGrath, J. Appl. Polym. Sci., Appl. Polym. Symp., 34, 103 (1987).

12. C. A. Pryde and M. Y. Hellman, J. Appl. Polym. Sci., 25, 2573 (1980).

13. C. O. Mork and D. B. Priddy, J. Appl. Polym. Sci., 45, 435 (1992).

14. A. J. Wnuk, T. F. Davidson, and J. E. McGrath, J. Appl. Polym. Sci., Appl. Polym. Symp., 34, 89 (1987).

15. S. Tsuge, T. Okumoto, Y. Sugimura, and T. Takeuchi, $J$. Chromatogr. Sci., 7, 253 (1969).

16. J. M. Challinor, J. Anal. Appl. Pyrolysis, 16, 323 (1989).

17. H. Ohtani, R. Fujii, and S. Tsuge, J. High Res. Chromatogr., 14, 388 (1991).

18. G. Holzer, T. F. Bourne, and W. Bertsch, J. Chromatogr., 468, 181 (1989)

19. K. B. Anderson and R. E. Winans, Anal. Chem., 63, 2901 (1991).

20. Y. Ishida, H. Ohtani, T. Kato, S. Tsuge, and T. Yano, Tappi J., 77, 177 (1994)

21. Y. Ishida, S. Isomura, S. Tsuge, H. Ohtani, T. Sekino, M. Nakanishi, and T. Kimoto, Analyst, 121, 853 (1996).

22. S. Teramachi, A. Takahashi, and I. Kagawa, Kogyo Kagaku Zasshi, 69, 685 (1966).

23. G. Sitaramaiah, J. Polym. Sci., Part A., 3, 2743 (1965).

24. S. Foti, M. Giuffrida, P. Maravigna, and G. Montaudo, J. Polym. Sci., Polym. Chem. Ed., 21, 1567 (1983).

25. A. Ballistreri, G. Montaudo, and E. Scamporrino, J. Polym. Sci., A, Polym. Chem., 26, 2113 (1988).

26. I. C. McNeill and A. Rincon, Polym. Deg. Stab., 31, 163 (1991)

27. G. Montaudo and C. Puglisi, Polym. Deg. Stab., 37, 91 (1992).

28. I. C. McNeill and A. Rincon, Polym. Deg. Stab., 39, 13 (1993).

29. A. D. Jorgensen, V. C. Stamoudis, and K. C. Pisel, Anal. Chem., 62, 683 (1990).

30. S. Honma, Ed., "Polycarbonate Resin Handbook," NikkanKogyo, Tokyo, 1992, p 32; Bayer AG., Jpn. Kokai Tokkyo Koho, JP58-84822 (1983), General Electric Co., Jpn. Kokai Tokkyo Koho, JP61-87724 (1986), and General Electric Co., Jpn. Kokai Tokkyo Koho, JP61-87725 (1986). 